\title{
Features of ovarian cancer in Lynch syndrome (Review)
}

\author{
KANAKO NAKAMURA, KOUJI BANNO, MEGUMI YANOKURA, MIHO IIDA, MASATAKA ADACHI, \\ KENTA MASUDA, ARISA UEKI, YUSUKE KOBAYASHI, HIROYUKI NOMURA, \\ AKIRA HIRASAWA, EIICHIRO TOMINAGA and DAISUKE AOKI
}

Department of Obstetrics and Gynecology, School of Medicine, Keio University, Tokyo 160-8582, Japan

Received March 14, 2014; Accepted May 30, 2014

DOI: $10.3892 / \mathrm{mco} .2014 .397$

\begin{abstract}
Lynch syndrome is a hereditary ovarian cancer with a prevalence of $0.9-2.7 \%$. Lynch syndrome accounts for $10-15 \%$ of hereditary ovarian cancers, while hereditary breast and ovarian cancer syndrome accounts for $65-75 \%$ of these cancers. The lifetime risk for ovarian cancer in families with Lynch syndrome is $\sim 8 \%$, which is lower than colorectal and endometrial cancers, and ovarian cancer is not listed in the Amsterdam Criteria II. More than half of sporadic ovarian cancers are diagnosed in stage III or IV, but $\geq 80 \%$ of ovarian cancers in Lynch syndrome are diagnosed in stage I or II. Ovarian cancers in Lynch syndrome mostly have non-serous histology and different properties from those of sporadic ovarian cancers. A screening method for ovarian cancers in Lynch syndrome has yet to be established and clinical studies of prophylactic administration of oral contraceptives are not available. However, molecular profiles at the genetic level indicate that ovarian cancer in Lynch syndrome has a more favorable prognosis than sporadic ovarian cancer. Inhibitors of the phosphatidylinositol 3-kinase/mammalian target of the rapamycin pathway and anti-epidermal growth factor antibodies may have efficacy for the disease. To the best of our knowledge, this is the first review focusing on ovarian cancer in Lynch syndrome.
\end{abstract}

\section{Contents}

1. Introduction

2. Etiology and diagnosis of Lynch syndrome

3. Characteristics of ovarian cancer in Lynch syndrome

4. Surveillance and prevention of ovarian cancer in Lynch syndrome

5. Chemoprevention of Lynch syndrome

Correspondence to: Dr Kouji Banno, Department of Obstetrics and Gynecology, School of Medicine, Keio University, Shinanomachi 35 Shinjuku-ku, Tokyo 160-8582, Japan

E-mail: kbanno@z7.keio.jp

Key words: lynch syndrome, ovarian cancer, surveillance, chemoprevention, risk-reducing surgery
6. Risk-reducing surgery for the prevention of Lynch syndrome

7. Genetics of ovarian cancer in Lynch syndrome

8. Conclusion

\section{Introduction}

Ovarian cancer is a gynecological malignancy with a poor prognosis. Signs and symptoms of ovarian cancer are less apparent in comparison with those in endometrial cancer, and early detection is difficult due to the anatomical location of the ovaries in the abdominal region. Early ovarian cancer often occurs in the abdominal area, but only $30 \%$ of cases are diagnosed in stage I or II and the majority of ovarian cancer is diagnosed at an advanced stage (1).

Ovarian cancer is conventionally viewed as familial, and epidemiologically the risk of development is 2- to 6-fold higher in females that have a first-degree relative with ovarian cancer, suggesting a strong link with their genetic background (2). Hereditary ovarian cancer may be classified into hereditary breast-ovarian cancer syndrome (including site-specific ovarian cancer and breast/ovarian cancer predisposition) and Lynch syndrome (3), while other pathogeneses account for $\leq 2 \%$ of hereditary ovarian cancer. Although breast cancer susceptibility gene 1 (BRCA1) and BRCA2, which have been identified as causative genes in hereditary breast-ovarian cancer, are involved in $65-75 \%$ of hereditary ovarian cancers, Lynch syndrome accounts for $10-15 \%$ of hereditary ovarian cancers (4). Lynch syndrome is an autosomal dominant hereditary cancer family syndrome that was previously referred to as hereditary non-polyposis colorectal cancer (HNPCC) (5). The present review focuses on the recent findings regarding the association between Lynch syndrome and hereditary ovarian cancer.

\section{Etiology and diagnosis of Lynch syndrome}

Patients with Lynch syndrome have high risks of familial endometrial cancer, urinary tract cancer, and small intestinal cancer. In 1999, the International Collaborative Group-HNPCC published the revised Amsterdam Criteria (AC) I as the international clinical criteria for Lynch syndrome (AC II) (Table I) (5). Lynch syndrome is mainly caused by germline mutations in DNA mismatch repair (MMR) genes. These MMR genes, including mutL homolog 1 (MLH1), mutS homolog 2 (MSH2), 
Table I. Clinical criteria for Lynch syndrome (HNPCC) (5).

Classic ICG-HNPCC Criteria (Amsterdam Criteria I, 1990)

There should be at least three relatives with colorectal cancer, and all the following criteria should be present.

i) One should be a first-degree relative of the other two.

ii) At least two successive generations should be affected.

iii) At least one colorectal cancer should be diagnosed before age 50.

iv) Familial adenomatous polyposis should be excluded.

v) Tumors should be verified by pathological examination.

Revised ICG-HNPCC Criteria (Amsterdam Criteria II, 1999)

There should be at least three relatives with a Lynch/HNPCC-associated cancer (cancer of the colorectum, endometrium, small bowel, ureter or renal pelvis).

i) One should be a first-degree relative of the other two.

ii) At least two successive generations should be affected.

iii) At least one of the relatives with cancers associated with HNPCC should be diagnosed before age 50 .

iv) Familial adenomatous polyposis should be excluded in the colorectal cancer case(s) if any.

v) Tumors should be verified by pathological examination.

ICG, International Collaborative Group; HNPCC, hereditary non-polyposis colorectal cancer.

Table II. Revised Bethesda Guidelines for Lynch syndrome (9).

Tumors from individuals should be tested for MSI in the following situations:

i) Colorectal cancer diagnosed in a patient who is $<50$ years of age.

ii) Presence of synchronous, metachronous colorectal, or other HNPCC-associated tumors ${ }^{\mathrm{a}}$, regardless of age.

iii) Colorectal cancer with the MSI-H histology diagnosed in a patient who is $<60$ years of age $\mathrm{e}^{\mathrm{b}}$.

iv) Colorectal cancer diagnosed in one or more first-degree relatives with an HNPCC-related tumor, with one of the cancers being diagnosed under age 50 years.

v) Colorectal cancer diagnosed in two or more first- or second-degree relatives with HNPCC-related tumors, regardless of age.

${ }^{a}$ LS-related tumors include colorectal, endometrial, gastric, ovarian, pancreatic, ureteral and renal pelvis, biliary tract, and brain (usually glioblastoma as observed in Turcot syndrome) tumors, sebaceous gland adenomas and keratoacanthomas in Muir-Torre syndrome, and carcinoma of the small bowel. 'Presence of tumor-infiltrating lymphocytes, Crohn's-like lymphocytic reaction, mucinous/signet-ring differentiation, or medullary growth pattern. HNPCC, hereditary non-polyposis colorectal cancer; MSI, microsatellite instability.

MSH3, MSH6, postmeiotic segregation increased 1 (PMS1) and PMS2, are tumor-suppressor genes involved in the repair of errors that occur during DNA replication (6). While mutations in MLH1 and MSH2 account for $90 \%$ of cases of Lynch syndrome, MSH6 and PMS2 mutations occur in in 7-10\% and $<5 \%$ of cases, respectively $(7,8)$. Patients with Lynch syndrome have a monoallelic germline mutation in one of these genes. When the other allele is somatically mutated, the two alleles are inactivated and normal expression of the MMR protein is lost. This causes a phenomenon referred to as microsatellite instability (MSI). Microsatellites are multiple tandem repeats of 1-6 nucleotides in the genome. MMR proteins repair abnormalities in microsatellite repeat numbers that occur during DNA replication. In cells without MMR proteins, this repair is not usually performed and MSI develops due to an accumulation of abnormal microsatellite repeats (6). This aberrant MMR system leads to the development of various types of cancer, including colorectal, endometrial, small intestinal, renal pelvis, ureteral, gastric and ovarian cancers. A definite diagnosis of Lynch syndrome requires the fulfillment of AC II or the Revised Bethesda Guidelines (Table II), high MSI or the abnormal immunostaining of MMR proteins and confirmation of a germline mutation of an MMR gene (9).

\section{Characteristics of ovarian cancer in Lynch syndrome}

Lynch syndrome has a prevalence of $0.9-2.7 \%$ and accounts for $10-15 \%$ of hereditary ovarian cancers (10). The lifetime risks and age at onset of Lynch syndrome-associated cancers are presented in Table III. The lifetime risk of ovarian cancer for females in families with Lynch syndrome is $8 \%$ (95\% confidence interval, 5.8-10.3), which is significantly higher than the $1.4 \%$ risk of ovarian cancer in the general population $(10,11)$. The age at onset of ovarian cancer in Lynch syndrome is $42-49$ years and that of sporadic ovarian cancer is 60-65 years (12-14). Although Lynch syndrome is diagnosed based on the germline mutations of MMR genes, $50 \%$ of cases are diagnosed at the onset of endometrial and ovarian cancer, 
Table III. Lifetime risks and age at onset in Lynch syndrome-associated cancers $(10,11)$.

\begin{tabular}{lccc}
\hline Cancer type & $\begin{array}{c}\text { General population } \\
\text { lifetime risk, } \%\end{array}$ & $\begin{array}{c}\text { Lynch/HNPCC } \\
\text { lifetime risk, \% }\end{array}$ & $\begin{array}{c}\text { Age at onset, } \\
\text { years }\end{array}$ \\
\hline Colon & 5.5 & $43-48$ & $44-61$ \\
Endometrium & 2.6 & $40-62$ & $27-72$ \\
Ovary & 1.4 & $5.8-10.3$ & $42-49$ \\
\hline
\end{tabular}

HNPCC, hereditary non-polyposis colorectal cancer.

as 'sentinel' cancers (15). This is clinically valuable in the identification of Lynch syndrome among young females with endometrial or ovarian cancer. However, the AC II criteria (Table I) do not include ovarian cancer as a sentinel cancer, and careful establishment of a family history by gynecologists or gynecological oncologists is required in these cases.

A study by Vasen et al (16) found a significantly higher lifetime risk of the development of ovarian cancer in $10.4 \%$ of MSH2 mutation carriers, compared with an $\sim 3$-fold lower risk of $3.4 \%$ in MLH1 mutation carriers $(\mathrm{P}=0.003)$. The study also reported a small difference in the mean age of onset between the MSH2 mutation carriers (45 years; range, 37-58 years) and the MLH1 mutation carriers (51 years; range, 35-75 years) (16). By contrast, the onset of ovarian cancer is also more frequent (33\%) in families with an MSH6 mutation, although the lifetime risk of this mutation has not been established (17). The majority of ovarian cancers in Lynch syndrome are well- or moderately-differentiated and at the International Federation of Gynecology and Obstetrics stage I or II at diagnosis. In a large-scale analysis of 80 patients registered between 1936 and 1997, 61\% of cases were at stage I, 23\% at stage II, $14 \%$ at stage III and $2 \%$ at stage IV; and number of the cases were early-stage ovarian cancer (14). Synchronous endometrial cancer was identified in $21.5 \%$ of these cases (14).

Sporadic ovarian tumors are pathologically subdivided into epithelial, gender cord stromal and germ cell tumors, with epithelial tumors being the most common. Among the epithelial tumors, high-grade serous carcinoma is the most common, and other subtypes include clear cell carcinoma (CCC), mucinous carcinoma and transitional cell carcinoma. In a retrospective study of ovarian cancer in Lynch syndrome, Watson et al (14) analyzed the clinical records of 79 patients with ovarian cancer from 11 countries. Of these patients, 44 were members of families with known Lynch syndrome mutations and the remaining patients had a family history corresponding to Lynch syndrome. Epithelial tumors were identified in 74 cases, including serous, mucous, endometrioid and mixed-type carcinomas and CCC. Non-epithelial ovarian tumors were also identified in 5 cases, and there were 2 cases each of granulosa cell, gender cord and endodermal sinus tumors and dysgerminoma. Immunohistochemical screening of MSH2, MLH1, MSH3, MSH6, PMS1 and PMS2 and MSI analysis was not performed. Thus, it cannot be concluded with certainty that these non-epithelial ovarian tumors were associated with Lynch syndrome.

Several studies, including the immunohistochemical examination and MSI analysis of MMR genes in ovarian cancer (18-23), have reported a wide variety of epithelial tumors associated with a high MSI status, such as malignant Müllerian mixed tumor, CCC, mucinous tumor, endometrioid tumor and mixed-type carcinomas. However, the association of pure high-grade serous carcinoma with high MSI caused by the germline mutation of MMR genes is unclear. In a large-scale study, Rosen et al (21) did not identify a case with high MSI among 168 cases of pure high-grade serous carcinoma. High-grade serous carcinoma is almost the sole histological type of hereditary ovarian cancer in hereditary breast-ovarian cancer syndrome with BRCA mutation $(24,25)$. For ovarian cancer caused by MMR mutation, Crijnen et al (26) found non-serous adenocarcinoma in seven of 19 cases (37\%) and Watson and Lynch (27) found this type in 31 of 48 cases (65\%). Thus, various histological types of ovarian cancer are caused by MMR mutation, while serous adenocarcinoma is the main histological type of ovarian cancer caused by BRCA mutation. This indicates that hereditary breast-ovarian cancer and ovarian cancer in Lynch syndrome may have different properties.

In an examination of prognosis, Grindedal et al (28) found that the 5-, 10-, 20- and 30-year survival rates of ovarian cancer in Lynch syndrome were 82.7, 80.6, 78 and 71.5\%, respectively. Crijnen et al (26) compared the prognoses of 26 patients with ovarian cancer and Lynch syndrome that fulfilled AC II criteria or had MMR mutations with those of 52 age- and stage-matched patients with sporadic ovarian cancer. The 5-year survival rates were 64.2 and $58.1 \%$, respectively, and they did not differ significantly $(\mathrm{P}=0.56)$. However, this may have been due to the similar effects of platinum-based chemotherapy and it was concluded that a further prospective study was required. Cancer cells with MMR mutations cannot undergo apoptosis in vitro and are resistan to platinum drugs $(29,30)$. However, an analysis of clinical data showed that the sensitivity of ovarian cancer with MMR gene mutations to platinum-based chemotherapy was similar to that of sporadic ovarian cancer (31). Various mechanisms may underlie resistance to platinum-based agents, including genetic or epigenetic changes of MMR genes, and further in vitro and in vivo studies are required.

\section{Surveillance and prevention of ovarian cancer in Lynch syndrome}

Appropriate methods for the surveillance of gynecological cancers in females of familes with a history of Lynch syndrome have not been fully established. The current guidelines are presented in Table IV (32). Annual endometrial sampling and transvaginal ultrasound in gynecological examinations are 
Table IV. Recommended management for at-risk members of families with Lynch syndrome (33).

Type of intervention Recommendation

Screening colonoscopy

Endometrial sampling

Transvaginal ultrasound for endometrial

and ovarian cancer

Urinalysis with cytology

History and examination with detailed review

of systems, education, and counseling

regarding Lynch syndrome

Colorectal resection

Hysterectomy or oophorectomy
Every 1-2 years beginning at age 20-25 years (age 30 years in MSH6 families), or 10 years younger than the youngest age at diagnosis in the family, whichever comes first

Every year beginning at age 30-35 years

Every year beginning at age $30-35$ years

Every 1-2 years beginning at age 25-35 years

Every year beginning at age 21 years

For persons with a diagnosed cancer or polyp not resectable by colonoscopy, subtotal colectomy favored with preferences of well-informed patient activity elicited

Discuss as an option after childbearing

MSH6, mutS homolog 6 .

recommended, although the level of evidence is not high. It is also unclear at what age screening for gynecological cancers should commence. This age should be determined based on the cumulative incidence of cancers in a family history of ovarian and endometrial cancers. In a review of retrospective studies, the ages of cancer onset and cumulative incidences in 90 families with Lynch syndrome, based on AC II criteria registered at the Royal Melbourne Hospital, were compared with those in the general population (33). The mean age at diagnosis of ovarian cancer was 48.3 years, and the cumulative incidences of the cancer were $0.2 \%$ at age $30,0.5 \%$ at 35 , and $0.7 \%$ at 40 years. Thus, effective screening for ovarian cancer is preferably commenced prior to age 30 , since the initiation of screening between age 30 and 35 would result in 3-7\% of gynecological cancers being overlooked.

Cancer antigen 125 (CA125) may be the most useful tumor marker for the detection of ovarian cancer. The CA125 tumor antigen is a glycoprotein found in the coelomic epithelium during the development of the majority of non-mucous ovarian cancers. The antigen is detected using a monoclonal antibody. One benefit of CA125 detection is that there is little elevation of the level at 10-60 months prior to the clinical diagnosis of ovarian cancer (34). A retrospective study conducted using the JANUS serum bank showed that half of the serum samples collected 18 months prior to the diagnosis of ovarian cancer had CA125 levels $>35 \mathrm{U} / \mathrm{ml}$ (the normal level), providing a sensitivity of $50 \%(34,35)$. In asymptomatic and postmenopausal females, the positive predictive value was $2 \%$ for detecting ovarian cancer using CA125 alone (36,37). Furthermore, in a large-scale ovarian cancer screening study of 22,000 subjects using CA125 alone, the sensitivity was $58 \%$ and the specificity was $98.5 \%$ (38). The specificity is extremely significant for ovarian cancer screening. The specificity of CA125 is limited as CA125 can be elevated in non-malignant and malignant diseases, including fibroid, endometriosis, menses, endometrial cancer and breast cancer, as well as other diseases, such as cirrhosis, congestive cardiac failure, diverticulitis and pancreatitis $(39,40)$. Thus, the CA125 level may provide a false-positive or -negative finding in screening for early detection and risk prediction of ovarian cancer in the general population, and thus is of limited practical utility. A combination of ultrasound and CA125 detection has also been found to be of limited value in ovarian cancer screening. Thus, the US Preventative Services Task Force indicated that routine screening for ovarian cancer with ultrasound, serum tumor markers or internal examination cannot be recommended, and that obtaining the best health care is the most practical approach $(37,41)$.

Although screening for ovarian cancer with ultrasound and CA125 may not be useful in the general population, it has been shown to be effective in the high-risk population with a BRCA1/2 mutation (42). BRCA mutation carriers are recommended to undergo screening with CA125 and transvaginal ultrasound twice a year, starting at age 30-35 or 5-20 years prior to the age at which a relative was diagnosed with ovarian cancer $(42,43)$. Although there is no consensus on the benefits of screening for ovarian cancer, zlarge-scale prospective trials exploring the benefits of screening for ovarian cancer in high-risk women are now in progress (44). There has been no clinical study with a focus on screening for hereditary ovarian cancer in females from families with Lynch syndrome and similar guidelines for these females have not been established. As aforementioned, the majority of ovarian cancers in Lynch syndrome are found at a relatively early stage and are frequently accompanied by endometrial cancer. Clarification of the pathology and clinical course is required to establish the optimal screening procedure for ovarian cancer in Lynch syndrome.

\section{Chemoprevention of Lynch syndrome}

The Concerted Action Polyp Prevention (CAPP2) trial was performed as a multinational collaborative prospective study of the chemoprevention of Lynch syndrome. Aspirin (600 mg/day) and resistant starch (30 g/day) were randomly administered and 
the chemopreventive effects on colorectal cancer were compared in MLH1 and MSH2 mutation carriers. The study concluded that aspirin reduced the incidence of colorectal cancer by $50 \%$ in females with Lynch syndrome (45). A CAPP3 study using a reduced dose of aspirin is planned. These results indicate that aspirin may also have a preventive effect for ovarian cancer in Lynch syndrome. Numerous case-control studies have investigated the chemoprevention of ovarian cancer in the general population, including use of Cancer and Steroid Hormone data collected by the Surveillance, Epidemiology, and End Results program. These results showed that the use of oral contraceptives reduces the development of endometrial and ovarian cancers by $50 \%(46,47)$. The effects of oral contraceptives on the prevention of ovarian cancer in Lynch syndrome are not clear, but their efficacy in females with a BRCA1/2 mutation, another high-risk population for ovarian cancer, indicates that oral contraceptives may reduce the incidence of Lynch syndrome-associated ovarian cancer (48-51). More studies are required to identify the efficacy of chemoprevention against Lynch syndrome-associated ovarian cancer.

\section{Risk-reducing surgery for the prevention of Lynch syndrome}

Risk-reducing gynecological surgery is another option for the prevention of ovarian cancer in Lynch syndrome. In 1997, the Cancer Genetics Studies Consortium reviewed evidence regarding prophylactic hysterectomy and bilateral salpingo-oophorectomy (BSO) for risk reduction, and published a consensus statement concluding that there was insufficient evidence to recommend that females with Lynch syndrome should undergo prophylactic surgery to reduce the risk of gynecological cancer (52). Despite this lack of evidence, hysterectomy with BSO has been indicated to be a reasonable preventive strategy for females with Lynch syndrome following completion of childbearing (53-55).

A study by Schmeler et al (56) provided evidence for the benefits of risk-reducing gynecological surgery in females with Lynch syndrome. A retrospective comparison was performed in 315 females with a documented germline mutation in MLH1, MSH2 or MSH6, including 61 females who had undergone prophylactic hysterectomy or had a benign disorder and had undergone hysterectomy with or without BSO, and 210 age-matched females who had not undergone this procedure. There were no cases of endometrial or ovarian cancer among the females who had undergone prophylactic surgery, but endometrial cancer developed in 69 females (33\%) and ovarian cancer in 12 females (5.5\%) among those who had not undergone prophylactic surgery. Females who had undergone prophylactic hysterectomy (61 females) and women who had undergone prophylactic BSO (47 females) were matched with mutation-positive women who had not undergone the procedure in question (210 females for the analysis of endometrial cancer and 223 for the analysis of ovarian cancer). Thus, risk-reducing surgery completely prevented new onset of endometrial and ovarian cancers in the cohort. The median age at diagnosis was 46 years for endometrial cancer and 42 years for ovarian cancer. These results are consistent with those obtained in previous studies of females with Lynch syndrome, with a mean age at diagnosis of 48-49 years for endometrial cancer $(57,58)$ and 42 years for ovarian cancer. These results support the performance of a risk-reducing hysterectomy with BSO in females with Lynch syndrome after the age of 35 or once childbearing is completed (56).

Lindor et al (32) discussed the recommendations for the care of individuals with an inherited predisposition to Lynch syndrome based on a review of the data and the opinions of specialists. In the study, risk-reducing hysterectomy and BSO was suggested for females at age $\geq 35$ years after childbearing, with hereditary counseling prior to surgery, including a discussion of the risks of the surgery, benefits and technical restrictions. Chen et al (59) compared three arms of annual gynecological examination, annual screening (transvaginal ultrasound + endometrial biopsy + CA125 level) and risk-reducing hysterectomy and BSO in a theoretical cohort of 10,000 females with Lynch syndrome to determine management strategies for preventing gynecological cancers. This analysis indicated that 75 surgeries would be required to save one life, in comparison with the screening arm. However, for cancer prevention, only 28 and 6 risk-reducing surgeries were required to prevent one case of ovarian and endometrial cancer, respectively. These results provide evidence that risk-reducing hysterectomy and BSO can reduce mortality from cancer and the incidence of cancer in females with Lynch syndrome.

The incidence of primary peritoneal cancer following risk-reducing $\mathrm{BSO}$ in females with a BRCA mutation is $0.8-1.0 \%(60,61)$. Primary peritoneal cancers have also been reported in females with Lynch syndrome who received risk-reducing BSO and long-term follow-up is required in these patients (62). In addition, females with Lynch syndrome are at a high risk of developing cancers metachronously or synchronously $(54,63)$. Once females with Lynch syndrome are affected with colorectal cancer, it is highly possible that they may develop endometrial or ovarian cancer. Similarly, in females with Lynch syndrome who are first diagnosed with endometrial or ovarian cancer, it is highly possible that they may develop colorectal cancer. In a study of 117 females with Lynch syndrome who developed dual cancers, Lu et al (55) identified 16 cases (14\%) of colon cancer and gynecological cancer (endometrial or ovarian cancer) that were diagnosed simultaneously. Of the remaining 101 women, 52 (51\%) with an initial diagnosis of endometrial or ovarian cancer, and $49(49 \%)$ with an initial diagnosis of colon cancer. In a similar study in 41 females (13\%), Schmeler et al (58) found a synchronous diagnosis of colon cancer and endometrial or ovarian cancer in three cases and metachronous diagnosis in 38 cases. Of these 41 cases, 21 (51\%) had gynecological cancer diagnosed following surgical treatment for colon cancer. Risk-reducing hysterectomy and BSO in these cases would have prevented gynecological cancer, which indicates that this risk-reduction surgery could be performed in females undergoing colorectal cancer surgery.

The disadvantages of risk-reducing hysterectomy and BSO include surgical complications and premature menopause. The common complications are bleeding, infection and injuries to the urinary tract and bowel. These complications have been found in 1-9\% of females with a benign disease following hysterectomy and BSO (56). In premenopausal females, risk-reducing BSO results in premature menopause, with symptoms including hot flashes, vaginal dryness, sexual dysfunction, sleep disturbance and an increased risk of osteo- 
porosis (56). A number of these conditions can be managed with hormonal or non-hormonal medications and there is no risk of uterine body cancer. Furthermore, an estrogen preparation can be used following hysterectomy and BSO. Parker et al (64) compared females who underwent oophorectomy at the time of hysterectomy for benign disease with females who underwent ovarian conservation. In the study, females undergoing oophorectomy prior to 55 years of age have an $8.58 \%$ excess mortality by age 80 , compared with $3.92 \%$ excess mortality in those undergoing oophorectomy prior to age 59. These findings do not necessarily apply to Lynch syndrome, and risk-reducing hysterectomy and BSO are reasonable options for females with Lynch syndrome, particularly those who are $>35$ years after childbearing. The risk-reduction for cancers, risks associated with surgery, side-effects and uncertainty of screening for gynecological cancers should be explained in patient counseling prior to surgery. Females undergoing colorectal cancer surgery should receive risk-reducing hysterectomy and BSO simultaneously.

\section{Genetics and epigenetics of ovarian cancer in Lynch syndrome}

Lynch syndrome-associated ovarian cancers mostly have non-serous histology and $\sim 82-84 \%$ are found in stage I or II, whereas only $30 \%$ of sporadic cancers are present in stage I or II (14). This aspect of ovarian cancer in Lynch syndrome is significantly different from findings for sporadic ovarian cancer and hereditary breast-ovarian cancer syndrome with BRCA1/2 mutation. In a recent comparison of patients with Lynch syndrome-associated ovarian cancer $(n=20)$ and sporadic ovarian cancer $(n=87)$, Niskakoski et al $(65)$ found differences in genetic and epigenetic mutations in the analysis of p53, KRAS/BRAF, phosphatidylinositol 3-kinase, catalytic subunit $\alpha$ (PIK3CA) and cyclin-dependent kinase inhibitor $2 \mathrm{~B}$ (CDKN2B) (tumor-suppressor genes), and long interspersed nucleotide element 1 (LINE1). PIK3CA is a cancer gene coding p110 $\alpha$, a catalytic subunit of PI3K (66). PIK3 and the mammalian target of rapamycin (mTOR) lie downstream of the Ras signaling pathway, which is activated in numerous tumors. The PIK3/mTOR pathway is directly activated by the mutation of PIK3CA and contributes to canceration. CDKN2B synthesizes a cyclin-dependent phosphoenzyme inhibitor (67) that forms a complex with CDK4 or CDK6 and inhibits the activation of CDK in G1 phase. Transcription of CDKN2B is activated by hypomethylation during canceration and CDKN2B in particular, plays an important role in the carcinogenesis of breast cancers. LINE1 is a retrotransposon with reverse transcriptase activity (68). LINE1 is usually methylated and inactivated, but can be demethylated and transcribed during canceration.

Niskakoski et al (65) examined mutations of p53, KRAS/BRAF and PIK3CA, and hypomethylation in CDKN2B and LINE1 in Lynch syndrome-associated ovarian cancer and sporadic ovarian cancer. In a clear contrast to sporadic cases, p53 and KRAS/BRAF mutations were absent in Lynch syndrome cases. The rates of p53 mutation differed significantly at $0 \%(0 / 20)$ in Lynch syndrome cases vs. $37 \%(32 / 87)$ in sporadic ovarian cancer $(\mathrm{P}<0.0001)$, and KRAS/BRAF mutations showed a similar trend of $0 \%(0 / 20)$ vs. $8 \%$ (7/87). Similar results have been found in colorectal cancer in Lynch syndrome (69). Among histological types of sporadic ovarian cancer, p53 mutations were found at a frequency of $85 \%(17 / 20)$ in serous adenocarcinoma and at significantly lower frequencies of $30 \%(8 / 27)$ in endometrioid adenocarcinoma $(\mathrm{P}=0.00029)$ and $18 \%(7 / 39)$ in clear cell adenocarcinoma $(\mathrm{P}<0.0001)$. PIK3CA mutations were found in $30 \%(6 / 20)$ of Lynch syndrome-associated cases of ovarian cancer, similar to the rates in endometrioid adenocarcinoma $(36 \% ; 10 / 28)$ and clear cell adenocarcinoma $(36 \% ; 14 / 39)$ in sporadic ovarian cancer. Among the histological types of sporadic ovarian cancer, PIK3CA mutation was significantly higher in endometrioid adenocarcinoma $(\mathrm{P}=0.013)$ and clear cell adenocarcinoma $(\mathrm{P}=0.011)$ compared with serous adenocarcinoma. mTOR inhibitors may be useful for the treatment of cases with a PIK3CA mutation (65). Hypomethylation of CDKN2B and LINE1 was significantly increased in sporadic ovarian cancers compared with Lynch syndrome cases (both $\mathrm{P}<0.0001)$. LINE1 is important in advanced stages of ovarian cancer (69) and this result is consistent with the favorable prognosis of ovarian cancer in Lynch syndrome.

\section{Conclusion}

There are few clinical studies on ovarian cancer in Lynch syndrome due to the small number of patients and relative lack of recognition of this disease. However, differences among histological types, stages at diagnosis and survival rates have been described. These findings indicate that ovarian cancer in Lynch syndrome has different properties from those of sporadic ovarian cancer and hereditary breast-ovarian cancer syndrome, which are other forms of hereditary ovarian cancer. The findings in Niskakoski et al (64) provide strong evidence for these differences. The absence of p53 and KRAS/BRAF mutations in ovarian cancer in Lynch syndrome is similar to the hereditary features of colorectal cancer in Lynch syndrome. Anti-epidermal growth factor antibodies may have efficacy for this form of colorectal cancer and may also be useful for ovarian cancer in Lynch syndrome (69). Cases with PIK3CA mutations may be treated effectively using mTOR inhibitors. Further clinical studies and investigation of the genetics of ovarian cancer in Lynch syndrome are required to improve risk assessment, screening and development of novel drugs for this disease.

\section{Acknowledgements}

The authors would like to thank Dr T. Fukushima and Dr S. Onomura for their helpful assistance. The authors gratefully acknowledge the grant support from the Japan Society for the Promotion of Science through a Grant-in-Aid for Scientific Research (KAKENHI), a Grant-in-Aid for Scientific Research (C) (grant no. 22591866), and a Grant-in-Aid for Young Scientists (B) (grant no. 24791718); the Medical Research Encouragement Prize of the Japan Medical Association; and the Keio Gijyuku Academic Development Fund. The funders had no role in data collection or the decision to publish.

\section{References}

1. Crispens MA: Endometrial and ovarian cancer in lynch syndrome. Clin Colon Rectal Surg 25: 97-102, 2012. 
2. Neesham D: Ovarian cancer screening. Aust Fam Physician 36 126-128, 2007.

3. Lu KH (ed): Clinical relevance of hereditary endometrial cancer In: Hereditary gynecologic cancer: risk, prevention, and management. 1st edition. Informa Healthcare, New York, NY, pp15-28, 2008.

4. Bewtra C, Watson P, Conway T, Read-Hippee C and Lynch HT: Hereditary ovarian cancer: a clinicopathological study. Int J Gynecol Pathol 11: 180-187, 1992.

5. Vasen HF, Watson P, Mecklin JP and Lynch HT: New clinical criteria for hereditary nonpolyposis colorectal cancer (HNPCC Lynch syndrome) proposed by the International Collaborative group on HNPCC. Gastroenterology 116: 1453-1456, 1999.

6. Jascur T and Boland CR: Structure and function of the components of the human DNA mismatch repair system. Int J Cancer 119: 2030-2035, 2006.

7. Harfe BD and Jinks-Robertson S: DNA mismatch repair and genetic instability. Annu Rev Genet 34: 359-399, 2000.

8. Kuiper RP, Vissers LE, Venkatachalam R, et al: Recurrence and variability of germline EPCAM deletions in Lynch syndrome. Hum Mutat 32: 407-414, 2011.

9. Umar A, Boland CR, Terdiman JP, et al: Revised Bethesda Guidelines for hereditary nonpolyposis colorectal cancer (Lynch syndrome) and microsatellite instability. J Natl Cancer Inst 96: 261-268, 2004

10. Aarnio M, Sankila R, Pukkala E, et al: Cancer risk in mutation carriers of DNA mismatch repair genes. Int J Cancer 81: 214-218, 1999.

11. Watson P, Vasen HF, Menklin JP, et al: The risk of extra-colonic, extra-endometrial cancer in the Lynch syndrome. Int $\mathrm{J}$ Cancer 123: 444-449, 2008.

12. Engel C, Loeffler M, Steinke V, et al: Risks of less common cancers in proven mutation carriers with lynch syndrome. J Clin Oncol 30: 4409-4415, 2012.

13. Ketabi Z, Bartuma K, Bernstein I, et al: Ovarian cancer linked to Lynch syndrome typically presents as early-onset, non-serous epithelial tumors. Gynecol Oncol 121: 462-465, 2011.

14. Watson P, Bützow R, Lynch HT, et al; International Collaborative Group on HNPCC: The clinical features of ovarian cancer in hereditary nonpolyposis colorectal cancer. Gynecol Oncol 82 223-228, 2001

15. Lu K: Gynecological malignancy as a 'sentinel cancer' for women with HNPCC. Gynecol Oncol 92: 421, 2004

16. Vasen HF, Stormorken A, Menko FH, et al: MSH2 mutation carriers are at higher risk of cancer than MLH1 mutation carriers: a study of hereditary nonpolyposis colorectal cancer families. J Clin Oncol 19: 4074-4080, 2001.

17. Cederquist K, Emanuelsson M, Wiklund F, Golovleva I, Palmqvist $\mathrm{R}$ and Grönberg H: Two Swedish founder MSH6 mutations, one nonsense and one missense, conferring high cumulative risk of Lynch syndrome. Clin Genet 68: 533-541, 2005.

18. Sood AK, Holmes R, Hendrix MJ and Buller RE: Application of the National Cancer Institute International criteria for determination of microsatellite instability in ovarian cancer. Cancer Res 61: 4371-4374, 2001.

19. Gras E, Catasus L, Argüelles R, et al: Microsatellite instability, MLH-1 promoter hypermethylation, and frameshift mutations at coding mononucleotide repeat microsatellites in ovarian tumors. Cancer 92: 2829-2836, 2001.

20. Cai KQ, Albarracin C, Rosen D, et al: Microsatellite instability and alteration of the expression of hMLH1 and hMSH2 in ovarian clear cell carcinoma. Hum Pathol 35: 552-559, 2004.

21. Rosen DG, Cai KQ, Luthra R and Liu J: Immunohistochemical staining of hMLH1 and hMSH2 reflects microsatellite instability status in ovarian carcinoma. Mod Pathol 19: 1414-1420, 2006.

22. Malander S, Rambech E, Kristoffersson U, et al: The contribution of the hereditary nonpolyposis colorectal cancer syndrome to the development of ovarian cancer. Gynecol Oncol 101: 238-243, 2006

23. Domanska K, Malander S, Måsbäck A and Nilbert M: Ovarian cancer at young age: the contribution of mismatch-repair defects in a population-based series of epithelial ovarian cancer before age 40. Int J Gynecol Cancer 17: 789-793, 2007.

24. Shaw PA, McLaughlin JR, Zweemer RP, et al: Histopathologic features of genetically determined ovarian cancer. Int J Gynecol Pathol 21: 407-411, 2002.

25. Piek JM, Torrenga B, Hermsen B, et al: Histopathological characteristics of BRCA1- and BRCA2-associated intraperitoneal cancer: a clinic-based study. Fam Cancer 2: 73-78, 2003.
26. Crijnen TE, Janssen-Heijnen ML, Gelderblom H, et al: Survival of patients with ovarian cancer due to a mismatch repair defect. Fam Cancer 4: 301-305, 2005.

27. Watson P and Lynch HT: Cancer risk in mismatch repair gene mutation carriers. Fam Cancer 1: 57-60, 2001.

28. Grindedal EM, Renkonen-Sinisao L, Vasen H, et al: Survival in women with MMR mutations and ovarian cancer: a multicentre study in Lynch syndrome kindreds. J Med Genet 47: 99-102, 2010

29. Aebi S, Kurdi-Haidar B, Gordon R, et al: Loss of DNA mismatch repair in acquired resistance to cisplatin. Cancer Res 56: 3087-3090, 1996.

30. Plumb JA, Strathdee G, Sludden J, Kaye SB and Brown R: Reversal of drug resistance in human tumor xenografts by 2'-deoxy-5-azacytidine-induced demethylation of the hMLHl gene promoter. Cancer Res 60: 6039-6044, 2000.

31. Helleman J, van Staveren IL, Dinjens WN, et al: Mismatch repair and treatment resistance in ovarian cancer. BMC Cancer 6: 201, 2006.

32. Lindor NM, Petersen GM, Hadley DW, et al: Recommendations for the care of individuals with an inherited predisposition to Lynch syndrome: a systematic review. JAMA 296: 1507-1517, 2006.

33. Brown GJ, St John DJ, Macrae FA and Aittomäki K: Cancer risk in young women at risk of hereditary nonpolyposis colorectal cancer: implications for gynecologic surveillance. Gynecol Oncol 80: 346-349, 2001.

34. Zurawski VR Jr, Orjaseter H, Anderson A and Jellum E: Elevated serum CA 125 levels prior to diagnosis of ovarian neoplasia: relevance for early detection of ovarian cancer. Int J Cancer 42: 677-680, 1988

35. Zurawski VR Jr, Sjovall K, Schoenfeld DA, et al: Prospective evaluation of serum CA 125 levels in a normal population, phase I: the specificities of single and serial determinations in testing for ovarian cancer. Gynecol Oncol 36: 299-305, 1990.

36. Skates SJ, Xu FJ, Yu YH, et al: Toward an optimal algorithm for ovarian cancer screening with longitudinal tumor markers. Cancer 76 (10 Suppl): 2004-2010, 1995.

37. Buys SS, Partridge E, Greene MH, et al; PLCO Project Team: Ovarian cancer screening in the Prostate, Lung, Colorectal and Ovarian (PLCO) cancer screening trial: findings from the initial screen of a randomized trial. Am J Obstet Gynecol 193: $1630-1639,2005$

38. Jacobs I, Davies AP, Bridges J, et al: Prevalence screening for ovarian cancer in postmenopausal women by CA 125 measurement and ultrasonography. BMJ 306: 1030-1034, 1993.

39. Bast RC Jr, Klug TL, St John E, et al: A radioimmunoassay using a monoclonal antibody to monitor the course of epithelial ovarian cancer. N Eng J Med 309: 883-887, 1983.

40. Jacobs IJ, Mackay J, Menon U, Skates SJ, Rosenthal AN and Fraser L: Familial ovarian screening - effective or ineffective? Br J Cancer 95: 1124, 1126-1127, 2006.

41. Partridge E, Kreimer AR, Buys S, et al: Ovarian cancer screening in the Prostate, Lung, Colorectal and Ovarian Screening Trial: Results from 4 years of annual screening in a randomized trial. Gynecol Oncol 104 (Suppl 3): abstr 10, S2-S35, 2007.

42. Burke W,Daly M, Garber J, et al: Recommendations for follow-up care of individuals with an inherited predisposition to cancer: II. BRCA1 and BRCA2. Cancer Genetics Studies Consortium. JAMA 227: 997-1003, 1997.

43. Armstrong K, Micco E, Carney A, Stopfer J and Putt M: Racial differences in the use of BRCA $1 / 2$ testing among women with a family history of breast or ovarian cancer. JAMA 293: 1729-1736, 2005.

44. Rosenthal AN, Fraser L, Manchanda R, et al: Results of annual screening in phase I of the United Kingdom familial ovarian cancer screening study highlight the need for strict adherence to screening schedule. J Clin Oncol 31: 49-57, 2013.

45. Burn J, Gerdes AM, Macrae F, et al; CAPP2 Investigators: Long-term effect of aspirin on cancer risk in carriers of hereditary colorectal cancer: an analysis from the CAPP2 randomised controlled trial. Lancet 378: 2081-2087, 2011

46. No authors listed: Oral contraceptive use and the risk of endometrial cancer. The Centers for Disease Control Cancer and steroid Hormone Study. JAMA 249: 1600-1604, 1983

47. No authors listed: The reduction in risk of ovarian cancer associated with oral-contraceptive use. The Cancer and Steroid Hormone Study for the Centers for Disease Control and the National Institute of Child Health and Human Development. N Engl J Med 316: 650-655, 1987.

48. Narod SA, Risch H, Moslehi R, et al: Oral contraceptives and the risk of hereditary ovarian cancer. Hereditary Ovarian Cancer Clinical Study Group. N Engl J Med 339: 424-428, 1998. 
49. Tavani A, Ricci E, La Vecchia C, et al: Influence of menstrual and reproductive factors on ovarian cancer risk in women with and without family history of breast or ovarian cancer. Int J Epidemiol 29: 799-802, 2000.

50. Whittemore AS, Balise RR, Pharoah PD, et al: Oral contraceptive use and ovarian cancer risk among carriers of BRCA1 or BRCA2 mutations. Br J Cancer 91: 1911-1915, 2004.

51. McLaughlin JR, Risch HA, Lubinski J, et al; Hereditary Ovarian Cancer Clinical Study Group: Reproductive risk factors for ovarian cancer in carriers of BRCA1 or BRCA 2 mutations: a case-control study. Lancet Oncol 8: 26-34, 2007.

52. Burke W, Petersen G, Lynch P, et al: Recommendations for follow-up care of individuals with an inherited predisposition to cancer. I. Hereditary nonpolyposis colon cancer. Cancer Genetics Studies Consortium. JAMA 277: 915-919, 1997.

53. Bertagnolli MM: Surgical prevention of cancer. J Clin Oncol 23: 324-332, 2005.

54. Lynch HT, Watson P, Shaw TG, et al: Clinical impact of molecular genetic diagnosis, genetic counseling, and management of hereditary cancer. Part II: Hereditary nonpolyposis colorecta carcinoma as a model. Cancer 86 (11 Suppl): 2457-2463, 1999.

55. Lu KH, Dinh M, Kolhmann W, et al: Gynecologic cancer as a 'sentinel cancer' for women with hereditary nonpolyposis colorectal cancer syndrome. Obstet Gynecol 105: 569-574, 2005.

56. Schmeler KM, Lynch HT, Chen LM, et al: Prophylactic surgery to reduce the risk of gynecologic cancers in the Lynch syndrome. N Engl J Med 354: 261-269, 2006.

57. Banno K, Kisu I, Yanokura M, et al: Epimutation and cancer: A new carcinogenic mechanism of Lynch syndrome (Review) Int J Oncol 41: 793-797, 2012.

58. Boks DE, Trujillo AP, Voogd AC, Morreau H, Kenter GG and Vasen HF: Survival analysis of endometrial carcinoma associated with hereditary nonpolyposis colorectal cancer. Int J Cancer 102: 198-200, 2002 .

59. Chen LM, Yang KY, Little SE, Cheung MK and Caughey AB: Gynecologic cancer prevention in Lynch syndrome/hereditary nonpolyposis colorectal cancer families. Obstet Gynecol 110: $18-25,2007$.

60. Kauff ND, Satagopan JM, Robson ME, et al: Risk-reducing salpingo-oophorectomy in women with a BRCA1 or BRCA2 mutation. N Engl J Med 346: 1609-1615, 2002.
61. Rebbeck TR, Lynch HT, Neuhausen SL, et al; Prevention and Observation of Surgical End Points Study Group: Prophylactic oophorectomy in carriers of BRCA1 or BRCA2 mutations. N Engl J Med 346: 1616-1622, 2002.

62. Schmeler KM, Daniels MS, Soliman PT, et al: Primary peritoneal cancer after bilateral salpingo-oophorectomy in two patients with Lynch syndrome. Obstet Gynecol 115: 432-434, 2010.

63. Lynch HT, Harris RE, Lynch PM, Guirgis HA, Lynch JF, Bardawil WA: Role of heredity in multiple primary cancer. Cancer 40 (4 Suppl): 1849-1854, 1977.

64. Parker WH, Broder MS, Liu Z, et al: Ovarian conservation at the time of hysterectomy for benign disease. Obstet Gynecol 106: 219-226, 2005.

65. Niskakoski A, Kaur S, Renkonen-Sinisalo L, et al: Distinct molecular profiles in Lynch syndrome-associated and sporadic ovarian carcinomas. Int J Cancer 133: 2596-2608, 2013.

66. Oda K: Targeting Ras-PI3K/mTOR pathway and predictive biomarkers in endometrial cancer. Gan To Kagaku Ryoho 38: 1084-1087, 2011 (In Japanese).

67. Costa-Guda J, Soong CP, Parekh VI, Agarwal SK and Arnold A: Germline and somatic mutations in cyclin-dependent kinase inhibitor genes CDKN1A, CDKN2B, and CDKN2C in sporadic parathyroid adenomas. Horm Cancer 4: 301-307, 2013.

68. Jürgens B, Schmitz-Dräger BJ and Schulz WA: Hypomethylation of L1 LINE sequences prevailing in human urothelial carcinoma. Cancer Res 56: 5698-5703, 1996.

69. Domingo E, Laiho P, Ollikainen M, et al: BRAF screening as a low-cost effective strategy for simplifying HNPCC genetic testing. J Med Genet 41: 664-668, 2004.

70. Woloszynska-Read A, Zhang W, Yu J, et al: Coordinated cancer germline antigen promoter and global DNA hypomethylation in ovarian cancer: association with the BORIS/CTCF expression ratio and advanced stage. Clin Cancer Res 17: 2170-2180, 2011. 